\title{
Microstructure and Interfacial Analysis of Multiferroic Tunnel Junction Interfaces
}

\author{
Sankara RK Sundaram*, Nagarajan Valanoor, ${ }^{*}$ Paul Munroe* \\ * School of Materials Science \& Engineering, University of New South Wales, NSW 2052, \\ Sydney, Australia.
}

In recent times multiferroic materials that simultaneously display ferroelectric and ferromagnetic ordering have generated considerable interest. The possible interaction between switchable fields is expected to result in additional functionalities and novel devices in which magnetization can be induced by electric field and the electric polarization can be switched by a magnetic field. ${ }^{[1],[2]}$ This phenomenon of co-existence, not only provides a rich platform for exciting research, but also will lead to novel devices in magnetic storage media and spintronics. ${ }^{[3],[4]}$ Bismuth ferrite (BFO) is one such material with a rhombohedral structure and a lattice constant $\left(\mathrm{a}_{\mathrm{r}}\right)$ of $0.396 \mathrm{~nm},{ }^{[5]}$ a ferroelectric Curie temperature of $820^{\circ} \mathrm{C}$ and an anti-ferromagnetic Neel temperature of $370{ }^{\circ} \mathrm{C}$. ${ }^{[6]}$ By combining BFO with doped manganites (half metallic perovskites in which the magnetic polarization arises from only one spin direction. $\left.{ }^{[7]}\right)$ such as lanthanum strontium manganese oxide $\left(\mathrm{La}_{0.7} \mathrm{Sr}_{0.3} \mathrm{MO}\right)$ an enhanced device performance is expected.

In the present investigation, epitaxial heterostructures consisting of LSMO and BFO were fabricated using state-of-art pulsed laser deposition (PLD). Strontium titanate (STO) (001) was used as the substrate to fabricate the heterostructure and a layer by layer deposition method was used. The PLD facility consists of a KrF laser source, load lock system for holding up to six different targets, a high temperature heating source and a high vacuum system. In the present study interfacial investigations were carried out for all the interfaces (STO-LSMO, STO-LSMO-BFO).

The LSMO bottom electrode layer was deposited under a vacuum of $100 \mathrm{mT}$, a deposition temperature of $900^{\circ} \mathrm{C}$ and laser energy of $\sim 1.8-2.0 \mathrm{~J} / \mathrm{cm}^{2}$. The laser repetition rate was about 20000 pulses at $7 \mathrm{~Hz}$ for a film thickness of $48 \mathrm{~nm}$. The BFO thin barrier layer was deposited at a vacuum of $2 \mathrm{mT}$ and a temperature of $850^{\circ} \mathrm{C}$ with a slightly lesser energy density. The laser repetition rate was varied between $7000(20 \mathrm{~nm})$ and $3000(12 \mathrm{~nm})$, as per the required film thickness. Interfacial investigations were carried out for samples with two different BFO (20 nm and $12 \mathrm{~nm}$ ) thicknesses, whilst the LSMO thickness was maintained at $\sim 50 \mathrm{~nm}$. The cooling rate (to room temperature) after the deposition was also varied to study the effect of cooling rate on diffusion at the interfaces. In Sample 1, an amphrous layer (room temperature deposition) of strontium ruthenium oxide (SRO) was deposited as a protective layer for the BFO film.

X-ray diffraction (XRD) studies of the films were used to confirm phase purity and the epitaxial growth of the film. Atomic force microscopy (AFM) was used to confirm the presence of step growth with a very smooth film surface.

Bright field TEM images of both Sample 1 and Sample 2 are shown in figure 1 and 2, respectively. The thickness of LSMO and BFO is closely matching the designed levels.

Scanning transmission electron microscope - electron dispersive spectroscopy (STEM-EDS) mapping was carried out for the following elemental peaks: Sr-K, Ti-K, La-L, Mn-K, Bi-L and $\mathrm{Fe}-\mathrm{K}$. The energy windows for Ti and La were adjusted in order to minimise the effect of overlapping elemental peaks. A STEM probe size of $1.5 \mathrm{~nm}$ was used. STEM-EDS data is 
shown in figure 3 and figure 4 for Samples 1 and 2, respectively. In both specimens it is evident that the STO-LSMO interfaces are free from any measureable chemical diffusion of elements across the interface. However, for both the LSMO-BFO interfaces it is evident that there is diffusion of Fe into the LSMO layer. The Fe diffusion takes place irrespective of the BFO film thickness, as well as the cooling rate used. This signifies a fundamental issue of chemical sharpness for such interfaces. The implications of the Fe diffusion to ferroelectricity of BFO thin films will be reported subsequently.

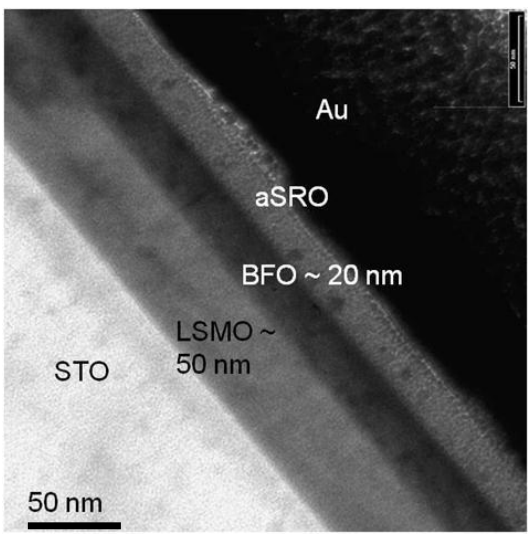

Figure1. BF TEM image of sample 1 (BFO $20 \mathrm{~nm}$, slow cooling)
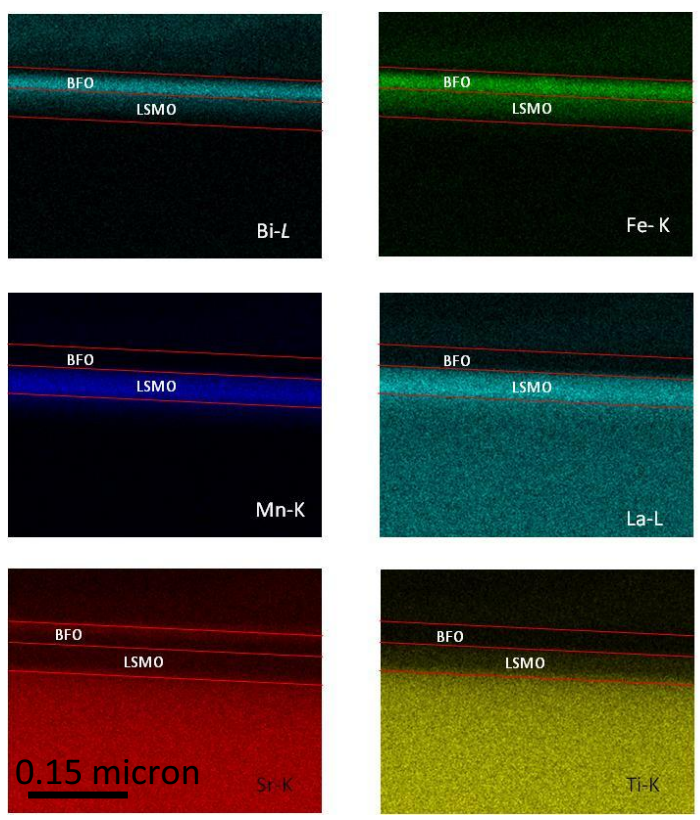

Figure. 3 STEM-EDS image of sample 1

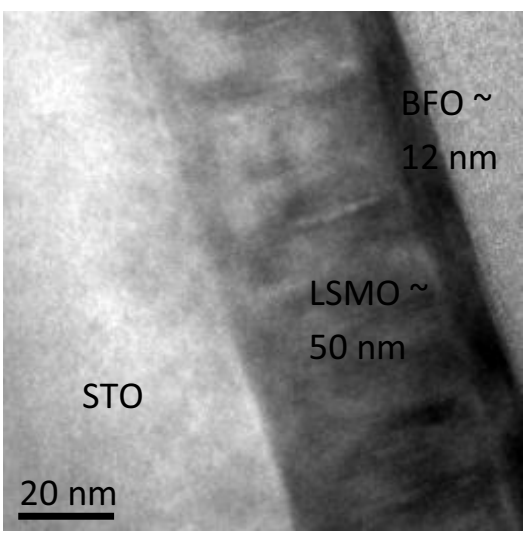

Figure2. BF TEM image of sample 2 (BFO $12 \mathrm{~nm}$, fast cooling)
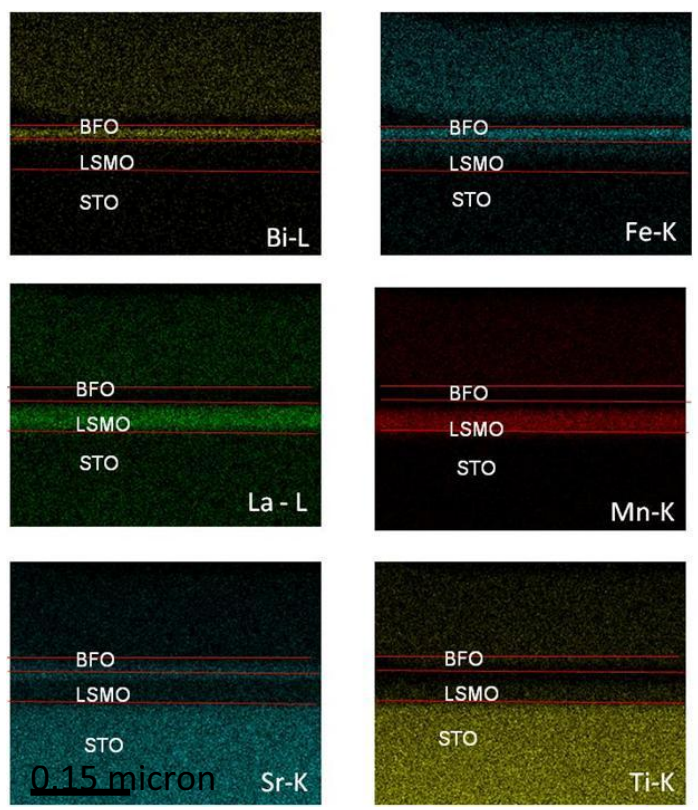

Figure. 4. STEM-EDS image of sample 2

References

1. Fiebig, M., et al., Observation of coupled magnetic and electric domains. Nature, 2002. 419(6909): p. 818-820.

2. Spaldin, N.A. and M. Fiebig, MATERIALS SCIENCE: The Renaissance of Magnetoelectric Multiferroics. Science, 2005. 309(5733): p. 391-392.

3. Béa, H., et al., Investigation on the origin of the magnetic moment of BiFeO3 thin films by advanced $x$-ray characterizations. Physical Review B, 2006. 74(2): p. 020101.

4. Seungbum, H., et al., Nanoscale piezoresponse studies of ferroelectric domains in epitaxial BiFeO[sub 3] nanostructures. Journal of Applied Physics, 2009. 105(6): p. 061619.

5. Ho Won, J., et al., Domain Engineering for Enhanced Ferroelectric Properties of Epitaxial (001) BiFeO Thin Films. Advanced Materials, 2009. 21(7): p. 817-823.

6. Martin, L.W., et al., Nanoscale Control of Exchange Bias with BiFeO3 Thin Films. Nano Letters, 2008. 8(7): p. 2050-2055.

7. Dorr, K., Ferromagnetic manganites: spin-polarized conduction versus competing interactions. Journal of Physics D: Applied Physics, 2006. 39(7): p. R125-R150. 